\title{
Permutations generated by a depth 2 stack and an infinite stack in series are algebraic
}

\author{
Murray Elder Geoffrey Lee \\ School of Mathematical and Physical Sciences \\ The University of Newcastle \\ Callaghan, New South Wales, 2308, Australia \\ murray.elder@newcastle.edu.au \\ geoffrey.a.lee@uon.edu.au

\section{Andrew Rechnitzer} \\ Department of Mathematics \\ University of British Columbia \\ Vancouver, British Columbia, V6T-1Z2, Canada \\ andrewr@math.ubc.ca
}

Submitted: Aug 1, 2014; Accepted: Apr 22, 2015; Published: Apr 29, 2015

Mathematics Subject Classifications: 05A05

\begin{abstract}
We prove that the class of permutations generated by passing an ordered sequence $12 \ldots n$ through a stack of depth 2 and an infinite stack in series is in bijection with an unambiguous context-free language, where a permutation of length $n$ is encoded by a string of length $3 n$. It follows that the sequence counting the number of permutations of each length has an algebraic generating function. We use the explicit context-free grammar to compute the generating function:

$$
\sum_{n \geqslant 0} c_{n} t^{n}=\frac{(1+q)\left(1+5 q-q^{2}-q^{3}-(1-q) \sqrt{\left(1-q^{2}\right)\left(1-4 q-q^{2}\right)}\right)}{8 q}
$$

where $c_{n}$ is the number of permutations of length $n$ that can be generated, and $q \equiv q(t)=\frac{1-2 t-\sqrt{1-4 t}}{2 t}$ is a simple variant of the Catalan generating function. This in turn implies that $c_{n}^{1 / n} \rightarrow 2+2 \sqrt{5}$.

Keywords: Pattern avoiding permutation; Algebraic generating function; Contextfree language
\end{abstract}




\section{Introduction}

Let $p=p_{1} p_{2} \ldots p_{n}$ and $q=q_{1} q_{2} \ldots q_{k}$ be permutations of length $n \geqslant k$. We say $p$ avoids $q$ if there are no $k$ indices $i_{1}<\cdots<i_{k}$ so that for all $s, t$,

$$
p_{i_{s}}<p_{i_{t}} \quad \text { if and only if } \quad q_{s}<q_{t} .
$$

For example, 25413 avoids 123 since it has no increasing subsequence of length 3.

Interest in sets of permutations that avoid a small set of "patterns" arose naturally in the study of stack-sorting (or equivalently stack-generating) algorithms. Knuth showed that a permutation $p$ can be generated by passing the ordered sequence $12 \ldots|p|$ through an infinite stack if and only if $p$ avoids 312, and that permutations of length $n$ avoiding 312 are counted by the Catalan numbers [15].

If $\mathfrak{q}$ is a list of permutations, let $A v_{n}(\mathfrak{q})$ be the set of permutations of length $n$ that avoid $q$ for each $q \in \mathfrak{q}$. We call $A v(\mathfrak{q})=\bigcup_{n=0}^{\infty} A v_{n}(\mathfrak{q})$ a pattern-avoidance class. A basis for a pattern avoidance class $A v(\mathfrak{q})$ is a set $\mathfrak{p}$ of pairwise avoiding permutations so that $A v(\mathfrak{p})=A v(\mathfrak{q})$. A class is finitely based if it is equal to $A v(\mathfrak{p})$ for $\mathfrak{p}$ finite.

The list of pattern-avoidance classes for which a generating function for the sequence counting $A v_{n}(\mathfrak{q})$ has been computed, or shown to be rational, algebraic or non-algebraic, is limited. Classes avoiding a single pattern of length 3 are enumerated by the Catalan numbers $[15,17]$ and so have an algebraic generating function. For length four, $A v(\{1342\})$ has an algebraic generating function $[8], A v(\{1234\})$ has a generating function that is Dfinite but not algebraic [13], and a closed form generating function for $A v(\{1324\})$ has not be found $[2,9]$. It is known that for any pattern $p$ of length four, $A v(\{p\})$ is in bijection with one of these three classes. For single patterns of length greater than four, and classes avoiding two or more patterns, various isolated results are known [4, 18].

In this article we consider the class of permutations generated by passing an ordered sequence through a stack of depth 2 and infinite stack is series, which was shown by the first author to have a finite basis consisting of 20 patterns [11]. The more general case of two infinite stacks in series has not been enumerated. Pierrot and Rossin recently proved a polynomial time algorithm to decide if a permutation can be sorted by two stacks in series [16]. A special case where both stacks are required to remain ordered was solved by Atkinson, Murphy and Ruškuc [7]. The number of permutations sortable by 2 stacks in parallel was recently solved by Albert and Bousquet-Mélou [3].

Several authors have considered the language-theoretic complexity of pattern avoidance classes — see for example [1, 5, 6, 10]. Atkinson, Livesey, and Tulley [6] showed that the set of permutations generated by passing an ordered sequence through a finite token-passing network is in bijection with a regular language. Initially we applied this technique to the finite network consisting of a stack of depth 2 followed by a stack of depth $k$ in series, constructing a sequence of languages and corresponding rational generating functions for small values of $k$. As $k$ increased, the rational generating functions appeared to converge to the algebraic function given in Theorem 11 below. However, this method does not constitute a proof. To prove the result we instead follow another path — we establish a bijection between permutations generated and an unambiguous 
context-free language. The generating function is then guaranteed to be algebraic by a well known theorem of Chomsky and Schützenberger.

The main work in this article is to establish the bijection with the context-free language. It has been suggested that the method employed to transform the relatively simple pushdown-automaton description of the language to the quartic generating function should be much easier than the method we detail here. We would welcome any insights into this - in our approach we merely apply the standard theory, and give the details for an interested reader.

\section{Establishing a bijection}

Let $\mathcal{P}$ be the set of permutations that can be generated by a stack of depth 2 and infinite stack in series, and fix $\rho, \lambda, \mu$ as the stack moves indicated in Figure 1.

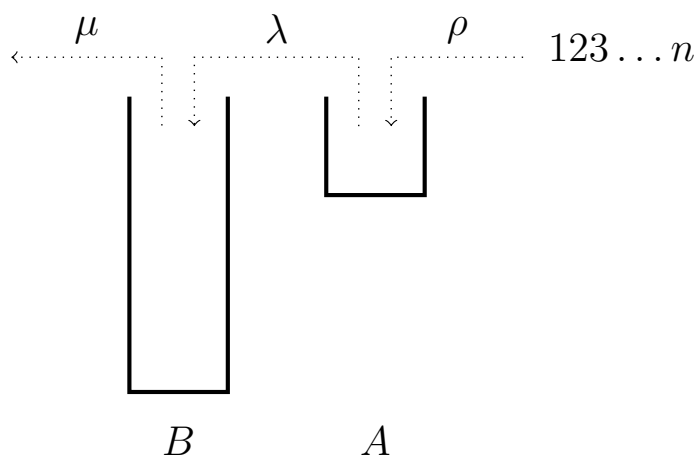

Figure 1: Token passing moves $\rho, \lambda$ and $\mu$ for two stacks in series.

Definition $1\left(\boldsymbol{D}_{\boldsymbol{a}, \boldsymbol{b}}(\boldsymbol{u})\right)$. If $u$ is a word over an alphabet that includes the letters $a$ and $b$, define $D_{a, b}(u)$ to be the number of $a$ letters minus the number of $b$ letters contained in $u$.

Definition $2\left(\mathcal{L}_{k, \infty}\right)$. Let $k \in \mathbb{N}$. The language $\mathcal{L}_{k, \infty}$ is the set of words $w \in\{\rho, \lambda, \mu\}^{*}$ satisfying

1. $D_{\rho, \lambda}(u) \in[0, k]$ and $D_{\lambda, \mu}(u) \in[0, \infty)$ for all prefixes, $u$, of $w$,

2. $D_{\rho, \lambda}(w)=D_{\lambda, \mu}(w)=0$.

Lemma 3. A word $w \in\{\rho, \lambda, \mu\}^{*}$ encodes a permutation in $\mathcal{P}$ if and only if $w \in \mathcal{L}_{2, \infty}$. Moreover, a word of length $3 n$ in $\mathcal{L}_{2, \infty}$ encodes a permutation of length $n$.

Proof. The first claim is clear from the definition. If $w \in \mathcal{L}_{2, \infty}$ has $n \rho$ letters, then $D_{\rho, \lambda}(w)=0$ implies $w$ has $n \lambda$ letters, and $D_{\lambda, \mu}(w)=0$ then implies $w$ has $n \mu$ letters, so the length of $w$ is $3 n$, and the number of tokens moves through the stacks is $n$. 
The language $\mathcal{L}_{2, \infty}$ consists of all possible ways to pass tokens through the system of stacks as in Figure 1. We wish to find a sublanguage that is in bijection with $\mathcal{P}$. From the set of all words in $\mathcal{L}_{2, \infty}$ that generate the same permutation, we will try to choose the string that outputs tokens as soon as possible, that is, has more $\mu$ letters closer to the front. The next definition will help to formalise this.

Definition 4 ( $\boldsymbol{\mu}$-ordering). Define an ordering, $\prec_{\mu}$, on words in $\{\rho, \lambda, \mu\}^{*}$ as follows. Let $\theta:\{\rho, \lambda, \mu\}^{*} \rightarrow\{\nu, \mu\}^{*}$ be a monoid homomorphism defined by $\theta(\mu)=\mu$ and $\theta(\rho)=$ $\theta(\lambda)=\nu$. If $u \neq v$ as strings then $u \prec_{\mu} v$ if $|u|=|v|$ and $\theta(u)$ precedes $\theta(v)$ in lexographic ordering on $\{\mu, \nu\}^{*}$ where $\mu<\nu$.

For example, if $u=\rho \lambda \mu \rho \lambda \mu$ and $v=\rho \lambda \rho \mu \lambda \mu$ then $u \prec_{\mu} v$. Note that both words generate the permutation 12 , and $u$ is obtained from $v$ by replacing the subword $\rho \mu$ by $\mu \rho$, which has no affect on the permutation being produced. More generally we have the following.

Lemma 5. Let $w \in \mathcal{L}_{2, \infty}$.

1. If $w=w_{0} \rho \mu w_{1}$ then $w^{\prime}=w_{0} \mu \rho w_{1}$ generates the same permutation as $w$, and $w^{\prime} \prec_{\mu} w$.

2. If $w=w_{0} \rho \lambda w_{1} \lambda \mu w_{2}$ with $D_{\rho, \lambda}\left(w_{0}\right)=1$ and $w_{1} \in \mathcal{L}_{1, \infty}$, then $w^{\prime}=w_{0} \lambda \rho w_{1} \mu \lambda w_{2}$ generates the same permutation as $w$, and $w^{\prime} \prec_{\mu} w$.

3. If $w=w_{0} \lambda \rho w_{1} \lambda \mu w_{2}$ with $D_{\rho, \lambda}\left(w_{0}\right)=1$ and $w_{1} \in \mathcal{L}_{1, \infty}$, then $w^{\prime}=w_{0} \rho \lambda w_{1} \mu \lambda w_{2}$ generates the same permutation as $w$, and $w^{\prime} \prec_{\mu} w$.

Proof. In each case it is clear that $w^{\prime} \prec_{\mu} w$. We must show that in each case the two strings generate the same permutation. For case (1) this is clear since $\rho$ and $\mu$ do not interact.

For case (2), since $D_{\rho, \lambda}\left(w_{0}\right)=1$, there must be one token (say $a$ ) left in the first stack after reading $w_{0}$, and since the next letter to be read is $\rho$, there must be one token (say b) ready to enter the first stack. See Figure 2 .

After reading $\rho \lambda, b$ moves to the top of stack $B$ and $a$ stays in stack $A$. Reading $w_{1}$ leaves $a$ and $b$ in place and outputs some permutation of input tokens. Finally $\lambda \mu$ outputs $a$, leaving $b$ on the top of stack $B$ and stack $A$ empty.

Starting from the initial configuration in Figure 2, the prefix $w_{0} \lambda \rho w_{1} \mu \lambda$ of $w^{\prime}$ moves $a$ to the top of stack $B$ and places $b$ in stack $A$. The permutation generated by $w_{1}$ is then passed across as before, then $a$ is output, and finally $b$ is moved to stack $B$, leaving the stacks in the same configuration as the prefix $w_{0} \rho \lambda w_{1} \lambda \mu$ of $w$.

A similar argument applies for Case (3) and is left to the reader.

Definition $6(\mathcal{L})$. The language $\mathcal{L}$ is the set of words $w \in \mathcal{L}_{2, \infty}$ that do not

1. contain a subword $\rho \mu$,

2. have a prefix $w_{0} \rho \lambda w_{1} \lambda \mu$ with $w_{1} \in \mathcal{L}_{1, \infty}$ and $D_{\rho, \lambda}\left(w_{0}\right)=1$,

3. have a prefix $w_{0} \lambda \rho w_{1} \lambda \mu$ with $w_{1} \in \mathcal{L}_{1, \infty}$ and $D_{\rho, \lambda}\left(w_{0}\right)=1$. 


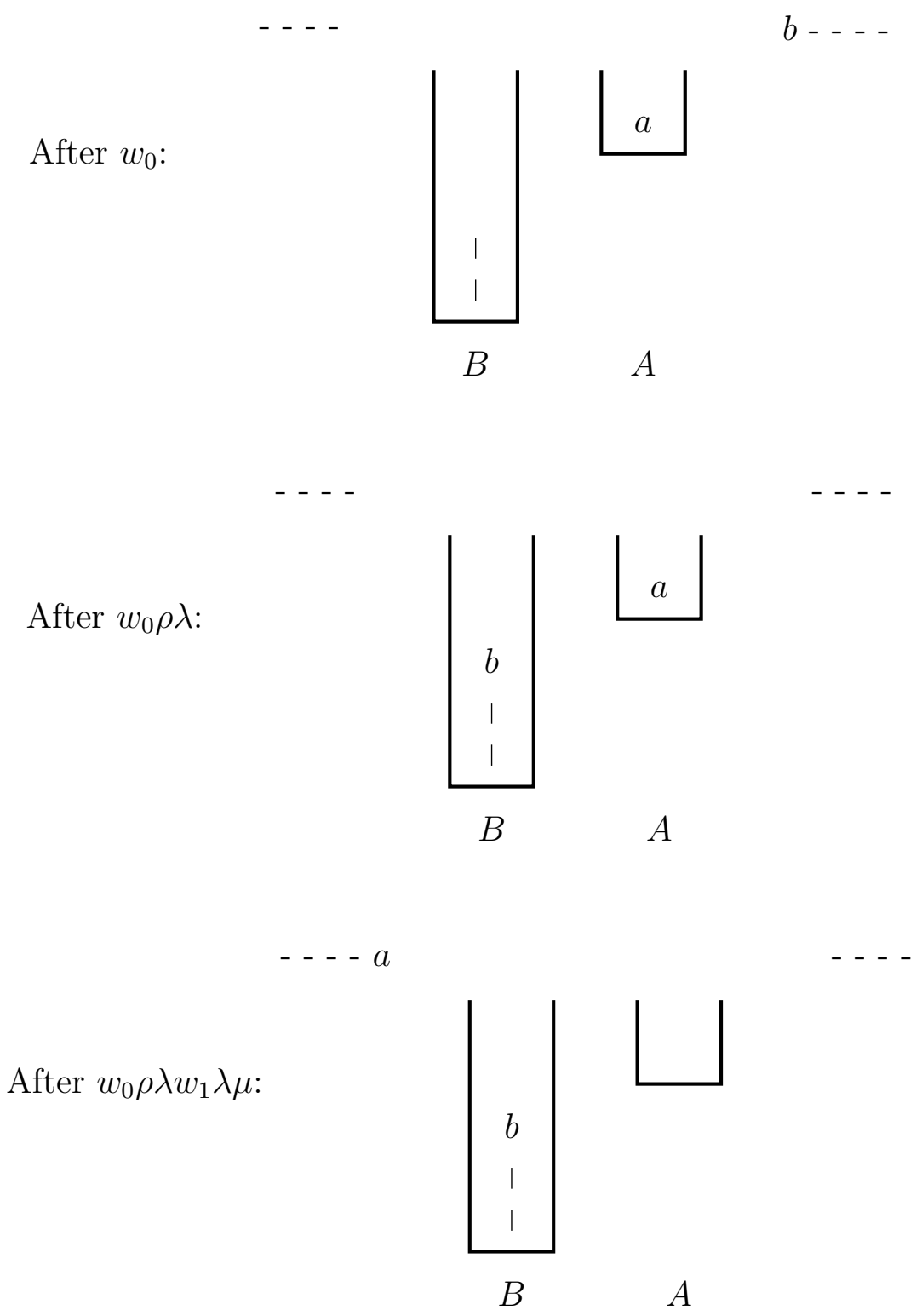

Figure 2: Stack configurations in the proof of Lemma 5.

Lemma 7. Let $w \in \mathcal{L}_{2, \infty}$. If either

1. $w=w_{0} \rho \lambda w_{1} \lambda w_{2} \mu w_{3}$ with $D_{\rho, \lambda}\left(w_{0}\right)=1, w_{1} \in \mathcal{L}_{1, \infty}$, and $w_{2} \in \mathcal{L}_{2, \infty}$ generates a permutation that avoids 312 , or

2. $w=w_{0} \lambda \rho w_{1} \lambda w_{2} \mu w_{3}$ with $D_{\rho, \lambda}\left(w_{0}\right)=1, w_{1} \in \mathcal{L}_{1, \infty}$, and $w_{2} \in \mathcal{L}_{2, \infty}$ generates a permutation that avoids 312 ,

then $w \notin \mathcal{L}$. 
Proof. Suppose for contradiction that $w \in \mathcal{L}, w=w_{0} v w_{1} \lambda w_{2} \mu w_{3}$ with $v \in\{\rho \lambda, \lambda \rho\}$, $D_{\rho, \lambda}\left(w_{0}\right)=1, w_{1} \in \mathcal{L}_{1, \infty}, w_{2}$ generates a permutation that avoids 312 , and moreover that $w_{0}$ is the longest prefix of $w$ with this property. That is, if $w=u_{0} v u_{1} \lambda u_{2} \mu u_{3}$ with $v \in\{\rho \lambda, \lambda \rho\}, D_{\rho, \lambda}\left(u_{0}\right)=1, u_{1} \in \mathcal{L}_{1, \infty}$ and $u_{2}$ generates a permutation that avoids 312 , then $\left|u_{0}\right| \leqslant\left|w_{0}\right|$.

Since $D_{\rho, \lambda}\left(w_{0} v w_{1}\right)=1$ and $\lambda$ moves a token from stack $A$ to stack $B$, after reading $w_{0} v w_{1} \lambda$ we have no tokens in stack $A$, and some token, say $a$, in stack $B$. See Figure 3 .

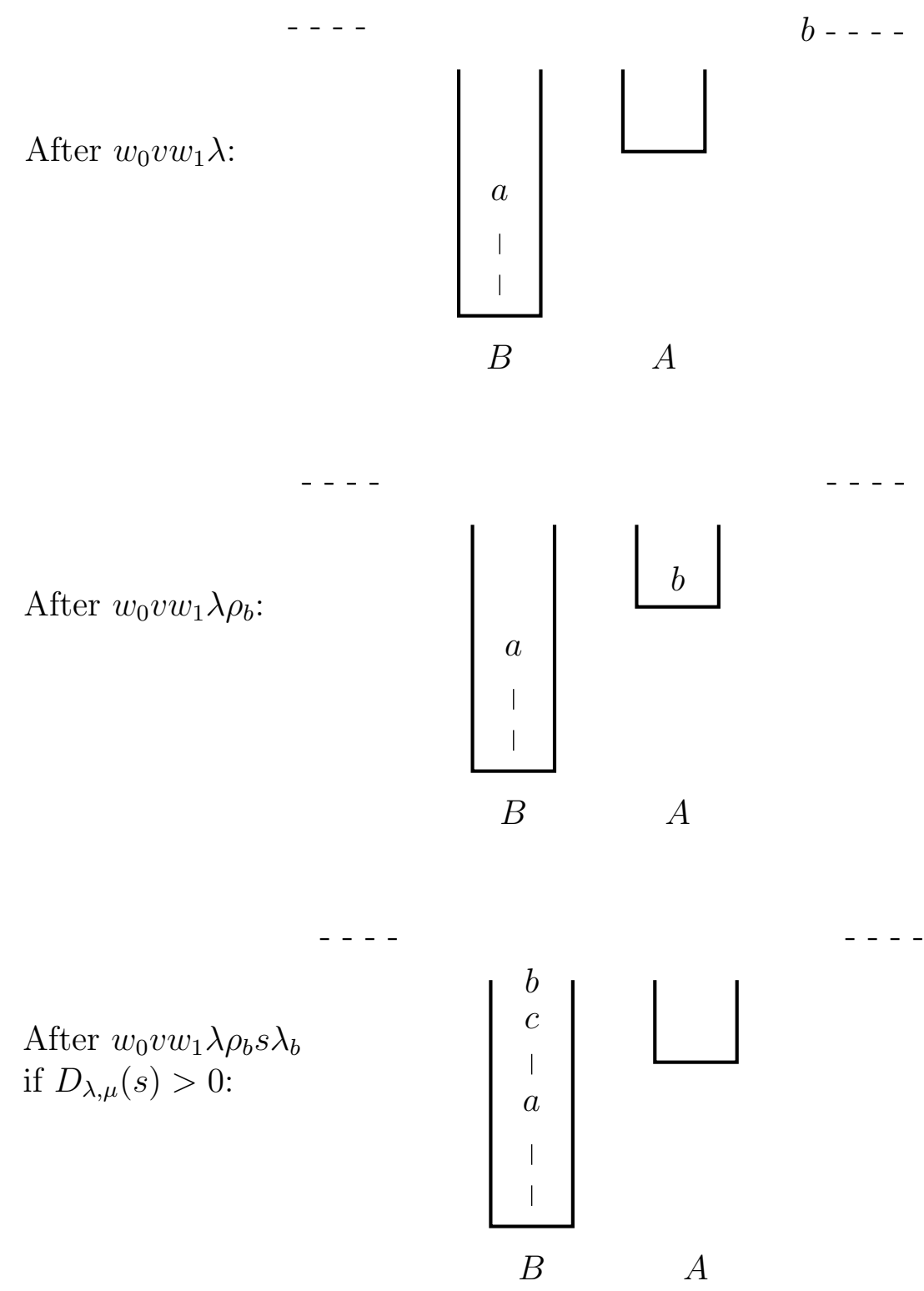

Figure 3: Stack configurations in the proof of Lemma 7.

Since $w \in \mathcal{L}, w_{2}$ cannot be empty, and since $w_{2}$ is a subword of $w \in \mathcal{L}$ we have 
$w_{2} \in \mathcal{L}$. So $w_{2}$ moves some sequence of tokens completely through the stacks, leaving $a$ in place. The first letter of $w_{2}$ must be $\rho$, which moves some token, say $b$, onto stack $A$. Let $\rho_{b}, \lambda_{b}, \mu_{b}$ be the letters in $w_{2}$ that correspond to moving $b$ through the stacks. Then $w_{2}$ has prefix $\rho_{b} s \lambda_{b} t \mu_{b}$ where $s, t$ are subwords.

Since stack $A$ contains $b$ while $s$ is read, if $\rho$ occurs in $s$ it must be immediately followed by $\lambda$, so $D_{\rho, \lambda}(u) \in[0,1]$ for all prefixes $u$ of $s$, and $D_{\rho, \lambda}(s)=0$. Further, if $D_{\lambda, \mu}(u)<0$ for any prefix $u$ of $s$, then $a$ would be output. Either $D_{\lambda, \mu}(s)=0$ (and $s \in \mathcal{L}$ ) or $D_{\lambda, \mu}(s)>0$.

If $s \in \mathcal{L}_{1, \infty}$ then $t \in \mathcal{L}_{2, \infty}$ and generates a permutation avoiding 312 since it is a subword of $w_{2}$. In this case $w$ has prefix $w=w_{0} v w_{1} \lambda \rho_{b} s \lambda_{b} t \mu_{b}$ with $D_{\lambda, \mu}\left(w_{0} v w_{1}\right)=1$ and $t$ generating a permutation avoiding 312 , which contradicts the choice of $w_{0}$ as the longest such prefix.

Therefore we must have $D_{\lambda, \mu}(s)>0$. In this case, after reading $s$ at least one token, say $c$, remains on top of $a$ in stack $B$ when $b$ is moved into it. After reading $\lambda_{b}$, the stack configuration is as in the third diagram shown in Figure 3.

Note that $a<b<c$ since they are input in this order. Let $\varepsilon$ denote the empty word. If $t \neq \varepsilon$ then it must contain at least one $\mu$ (it cannot leave a token covering $b$, and cannot just be $\rho$ or $\rho \rho)$ so it moves a token $d>c$ to the output. This means $w_{2}$ generates the subpermutation $d b c$ which is order equivalent to 312 , contradicting our assumption. Thus $t=\varepsilon$ and $w_{2}$ has prefix $\rho_{b} s \lambda_{b} \mu_{b}$, with $s \in\{\rho \lambda, \mu\}^{*}$. Either $s$ ends with $\rho \lambda$, or $s=u \rho \lambda s^{\prime}$ where $D_{\lambda, \mu}(u)=D_{\lambda, \mu}(s)$ since $D_{\lambda, \mu}$ starts at zero and increases to this value. Thus $s^{\prime} \in \mathcal{L}$, and $w=w_{0} v w_{1} \lambda \rho_{b} u \rho \lambda s^{\prime} \lambda_{b} \mu_{b}$ with $D_{\rho, \lambda}\left(w_{0} v w_{1} \lambda \rho_{b} u\right)=1$, which contradicts $w \in \mathcal{L}$.

Theorem 8. There is a bijection between permutations in $\mathcal{P}$ of length $n$ and words in $\mathcal{L}$ of length $3 n$.

Proof. Consider the map that sends a word of length $3 n$ in $\mathcal{L} \subseteq \mathcal{L}_{2, \infty}$ to the permutation of length $n$ it generates. If $\sigma \in \mathcal{P}$ then there is some word $w \in \mathcal{L}_{2, \infty}$ that generates it by Lemma 3. If $w \notin \mathcal{L}$, then $w$ must either contain $\rho \mu$, or have prefix $w_{0} \rho \lambda w_{1} \lambda \mu$ or $w_{0} \lambda \rho w_{1} \lambda \mu$ with $D_{\rho, \lambda}\left(w_{0}\right)=1$ and $w_{1} \in \mathcal{L}_{1, \infty}$. We rewrite $w$ as follows.

While $w$ contains $\rho \mu$ or has prefix $w_{0} \rho \lambda w_{1} \lambda \mu$ or $w_{0} \lambda \rho w_{1} \lambda \mu$ :

1. Replace $\rho \mu$ with $\mu \rho$

2. Replace $w_{0} \rho \lambda w_{1} \lambda \mu$ with $w_{0} \lambda \rho w_{1} \mu \lambda$

3. Replace $w_{0} \lambda \rho w_{1} \lambda \mu$ with $w_{0} \rho \lambda w_{1} \mu \lambda$

Each iteration replaces the current word by a word which generates the same permutation and is shorter in the $\mu$-ordering by Lemma 5 , so the procedure must terminate (there are finitely many words less than $w$ in the $\mu$-ordering). It follows that the map is surjective. We complete the proof by showing it is injective.

Suppose we have two words $u, v \in \mathcal{L}$ that generate the same permutation, and that $u \neq v$ as strings. Write

$$
u=u_{1} u_{2} \ldots u_{n} \text { and } v=v_{1} v_{2} \ldots v_{n}
$$


where $u_{i}, v_{i} \in\{\rho, \lambda, \mu\}$.

Since $u, v \in \mathcal{L}$ we have $u_{1}=v_{1}=\rho$. Let $k \in[2, n]$ be such that $u_{i}=v_{i}$ for $i<k$ and $u_{k} \neq v_{k}$. Let $z=u_{1} \ldots u_{k-1}=v_{1} \ldots v_{k-1}$, so

$$
u=z u_{k} \ldots u_{n} \text { and } v=z v_{k} \ldots v_{n} .
$$

First consider the case that one of $u_{k}, v_{k}$ is $\mu$. Without loss of generality assume $u=z \mu u_{k+1} \ldots u_{n}$. Then $z$ must leave some token, say $a$, at the top of stack $B$, and $u_{k}=\mu$ outputs this token.

If $v_{k}=\lambda$, then $a$ will be covered and $v$ will not be able to generate the same permutation. So we must have $v_{k}=\rho$. Then $v_{k+1} \neq \mu$. If $v_{k+1}=\lambda$ then $a$ is covered. So $v_{k+1}=\rho$. Then $v_{k+2} \neq \mu$, if $v_{k+2}=\lambda$ then $a$ is covered, and $v_{k+2} \neq \rho$ since stack $A$ contains two tokens. So we have a contradiction, and it follows that neither $u_{k}, v_{k}$ can be $\mu$.

Without loss of generality assume $u_{k}=\rho$ and $v_{k}=\lambda$. Then $z$ must leave at least one token in stack $A$ to be followed by $\lambda$, and at most one token in stack $A$ to be followed by $\rho$. Let $a$ be the token in $A$, and $b$ the token moved from the input by $u_{k}=\rho$. See Figure 4. Note that we have $D_{\rho, \lambda}(z)=1$.

In $u, z \rho$ must be followed by $\lambda$ since stack $A$ is full after the $\rho$ and $\rho$ cannot be followed by a $\mu$. So $u$ has prefix $z \rho \lambda$ and we have the configuration shown in the second diagram in Figure 4.

In $v, z \lambda$ can be followed by either $\mu$ or $\rho$ but not $\lambda$ since stack $A$ is empty after $v_{k}=\lambda$. Suppose $v_{k+1}=\mu$. Then after reading $z \lambda \mu$ we have the configuration shown in the third diagram in Figure 4. Since $u$ and $v$ are assumed to produce the same permutation, the next $\mu$ letter appearing in $u$ after the prefix $z \rho \lambda$ must move $a$ to the output. Let $\lambda_{a}, \mu_{a}$ be the letters in $u$ that move the token $a$. Then $u$ has prefix $z \rho \lambda \kappa_{1} \lambda_{a} \kappa_{2} \mu_{a}$ where $\kappa_{1}, \kappa_{2} \in\{\rho, \lambda\}^{*}$. The subword $\kappa_{2}$ cannot move tokens to cover $a$ in stack $B$, so cannot contain any $\lambda$ letters, and cannot contain any $\rho$ letters since it is followed by $\mu$, so it must be empty. The subword $\kappa_{1}$ must be of the form $(\rho \lambda)^{i}$ for $i \geqslant 0$, since it cannot move $a$. So $u$ has prefix $z(\rho \lambda)^{i} \rho \lambda \lambda_{a} \mu_{a}$ with $D_{\rho, \lambda}\left(z(\rho \lambda)^{i}\right)=1$, so $u \notin \mathcal{L}$.

It follows that $v_{k+1}=\rho$, so we have

$$
u=z \rho \lambda u_{k+2} \ldots u_{n}, v=z \lambda \rho v_{k+2} \ldots v_{n} .
$$

The two configurations of the stacks after reading the length $k+1$ prefixes of $u$ and $v$ respectively are shown in Figure 5.

We now consider two possibilities: either $a$ precedes $b$ in the permutation generated by $u$ and $v$, or $b$ precedes $a$.

\section{Case 1: $a$ precedes $b$}

Mark the letters $\lambda, \mu$ in $u$ and $v$ that correspond to moving the token $a$ by appending the subscript $a$. So $u$ has prefix $z \rho \lambda w_{1} \lambda_{a} w_{2} \mu_{a}$ and $v$ has prefix $z \lambda_{a} \rho w \mu_{a}$ where $w, w_{1}, w_{2} \in$ $\{\rho, \lambda, \mu\}^{*}$.

First consider the word $v$. Since $w$ cannot end with $\rho$, and $b$ must remain in stack $A$ until $a$ is output, and $w$ cannot leave any tokens covering $a$ in stack $B$, and we have 


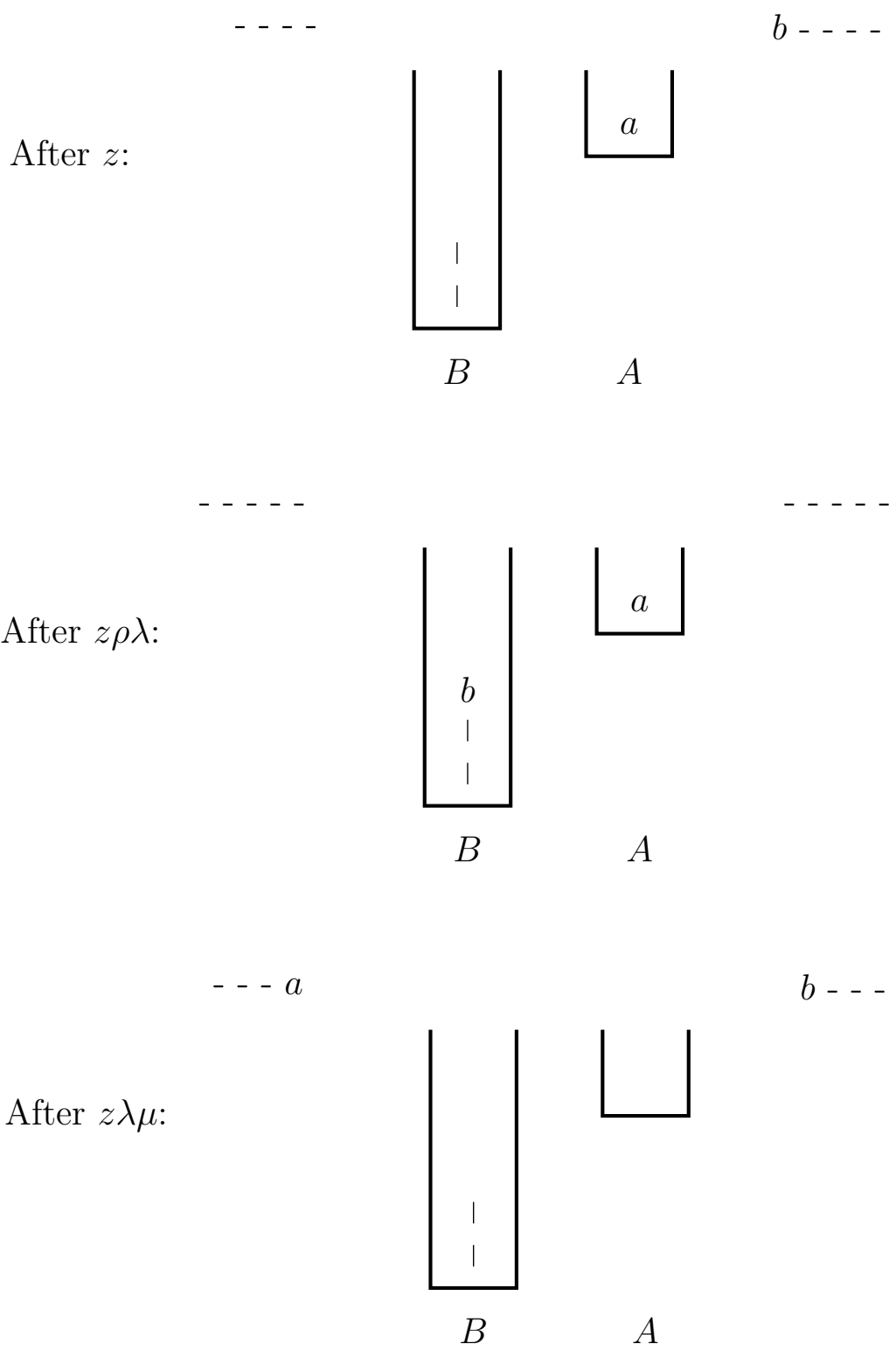

Figure 4: Stack configurations in Theorem 8 where $u_{k}=\rho$ and $v_{k}=\lambda$.

$w \in \mathcal{L}_{1, \infty}$. If $w$ is empty then $v$ contains $\rho \mu_{a}$ which means $v \notin \mathcal{L}$. Thus $w$ is nonempty, so moves some tokens, say $t_{1}, \ldots, t_{s}$, from the input to the output.

Since $u$ generates the same permutation as $v$, it must also move the tokens $t_{1}, \ldots, t_{s}$ through the stacks and output them before $a$ is output. The subword $w_{1}$ cannot leave any tokens covering $a$ in stack $A$, so $w_{1} \in\{\rho \lambda, \mu\}^{*}$. If $w_{1}$ leaves some token $t_{i}$ in stack $B$, then $\lambda_{a}$ will cover it by $a$, which means $t_{i}$ will appear after $a$ in the output, so $u$ does not generate the same permutation as $v$. Thus after $w_{1}$ the stacks are the same as before $w_{1}$, so $w_{1} \in \mathcal{L}_{1, \infty}$. Let $t_{1}, \ldots t_{r}$ with $r \leqslant s$ be the tokens moved to the output by $w_{1}$. The 


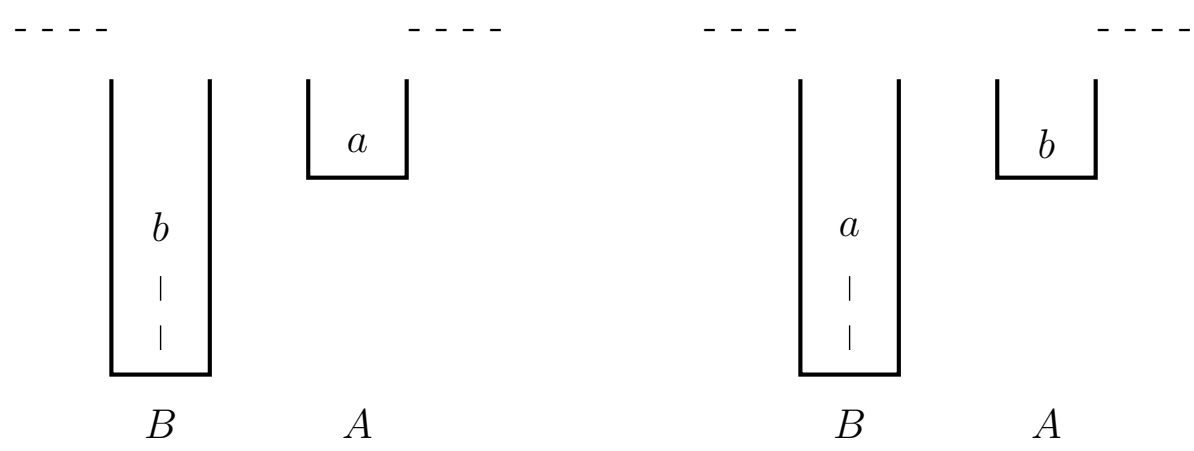

Figure 5: Stack configurations after $z \rho \lambda$ and $z \lambda \rho$ in Theorem 8.

situtation after reading $w_{1}$ is shown in Figure 6.

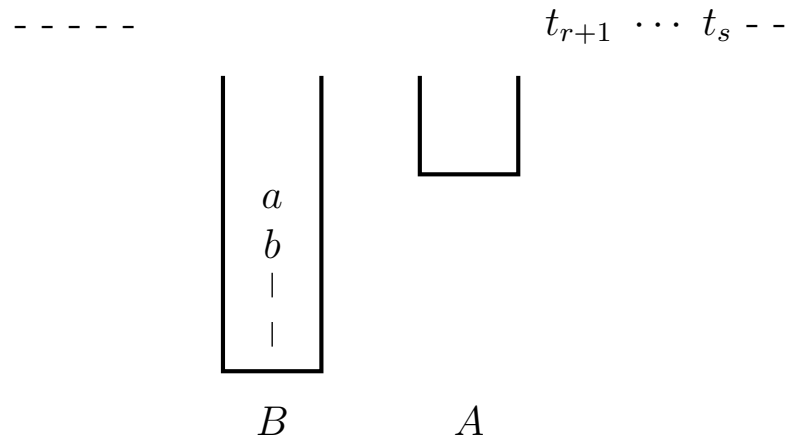

Figure 6: Stack configuration after $z \rho \lambda w_{1} \lambda_{a}$ in Case 1 in Theorem 8.

If $w_{2}$ is empty then $u$ has prefix $z \rho \lambda w_{1} \lambda_{a} \mu_{a}$ with $w_{1} \in \mathcal{L}_{1, \infty}$ which is forbidden, so $w_{2}$ must move some tokens. Also $w_{2}$ cannot leave any tokens in stack $B$. Either $w_{2}$ leaves some tokens in stack $A$, or not.

If $w_{2}$ leaves a token in stack $A$, this token cannot be one of $t_{r+1}, \ldots, t_{s}$ or else $u$ would generate a different permutation to $v$. Therefore this token is moved into stack $A$ after $t_{r}$ by a letter $\rho$. This letter cannot be followed by $\mu$, and since it remains in stack $A$ it is not followed by $\lambda$. So this letter is either the last letter of $w_{2}$, or is followed by another $\rho$, which must also remain in stack $A$. Thus $w_{2}$ ends with $\rho$, but this is a contradiction since $w_{2}$ is followed by $\mu_{a}$.

Thus $w_{2}$ does not leave any tokens in stacks $A$ or $B$, so moves $t_{r+1}, \ldots, t_{s}$ from the input to the output, so $w_{2} \in \mathcal{L}_{2, \infty}$. Note that $w_{1} w_{2}$ produces the same permutation of $t_{1}, \ldots, t_{s}$ as $w$ does, and $w \in \mathcal{L}_{1, \infty}$ so generates a 312 -avoiding permutation of $t_{1}, \ldots, t_{s}$. The subword $w_{1}$ permutes the first $r$ tokens, and so $w_{2}$ must produce a permutation of $t_{r+1}, \ldots, t_{s}$ that avoids 312 . In this case $u$ has prefix $z \rho \lambda w_{1} \lambda_{a} w_{2} \mu_{a}$ where $D_{\rho, \lambda}(z \rho \lambda)=1$, 
$w_{1} \in \mathcal{L}_{1, \infty}$ and $w_{2}$ generates a 312 -avoider, so by Lemma $7 u$ must also contain a prefix that is not allowed if $u \in \mathcal{L}$. This is a contradiction, so this case does not apply.

\section{Case 2: $b$ precedes $a$}

We return to the situation shown in Figure 5 with $u=z \rho \lambda u_{k+2} \ldots u_{n}$ and $v=$ $z \lambda \rho v_{k+2} \ldots v_{n}$. Mark the letters $\rho, \lambda, \mu$ in $u$ and $v$ that correspond to moving the token $b$ by appending a subscript $b$. Then $u$ has prefix $z \rho_{b} \lambda_{b} w \mu_{b}$ and $v$ has prefix $z \lambda \rho_{b} w_{1} \lambda_{b} w_{2} \mu_{b}$ where $w, w_{1}, w_{2} \in\{\rho, \lambda, \mu\}^{*}$.

First consider the word $u$. Since $w$ is followed immediately by $\mu_{a}, w$ cannot end with a $\rho$, and since $a$ must remain in stack $A$ until $b$ is output and $w$ cannot leave any tokens covering $b$ in stack $B$, we have $w \in \mathcal{L}_{1, \infty}$.

If $w$ is empty then $u$ has prefix $z \rho_{b} \lambda_{b} \mu_{b}$. For $v$ to produce the same permutation the next token it must output from the configuration shown in Figure 5 is $b$, so $w_{1} \in$ $\{\rho \lambda\}^{*}$ and $w_{2} \in\{\rho\}^{*}$. Since $w_{2}$ is followed by $\mu_{b}$ then $w_{2}$ must be empty. Then $v$ has prefix $z \lambda \rho_{b}(\rho \lambda)^{i} \lambda_{b} \mu_{b}$ for some $i \geqslant 0$. If $i=0$ then $v$ has prefix $z \lambda \rho_{b} \lambda_{b} \mu_{b}$ with $D_{\rho, \lambda}(z)=1$ which violates the third condition of Definition 6 , and if $i>0$ then $v$ has prefix $\left(z \lambda \rho_{b}(\rho \lambda)^{i-1}\right) \rho \lambda \lambda_{b} \mu_{b}$ with $D_{\rho, \lambda}\left(z \lambda \rho_{b}(\rho \lambda)^{i-1}\right)=1$ which violates the second condition of Definition 6 , so $v \notin \mathcal{L}$.

Thus $w$ is nonempty, so moves some tokens, say $t_{1}, \ldots, t_{s}$, from the input to the output. Since $v$ generates the same permutation as $u$, it must also move the tokens $t_{1}, \ldots, t_{s}$ through the stacks and output them before $b$ is output. The subword $w_{1}$ cannot leave any tokens covering $b$ in stack $A$, so $w_{1} \in\{\rho \lambda, \mu\}^{*}$.

If $w_{1}$ leaves some token $t_{i}$ in stack $B$, then $\lambda_{b}$ will cover $t_{i}$ with $b$ so $t_{i}$ will appear after $b$ in the permutation generated by $v$, which is different to the permutation generated by $u$. Thus after $w_{1}$ the stacks are the same as before $w_{1}$, so $w_{1} \in \mathcal{L}_{1, \infty}$. Let $t_{1}, \ldots t_{r}$ with $r \leqslant s$ be the tokens moved to the output by $w_{1}$. The situation is shown in Figure 7.



Figure 7: Stack configuration after $z \lambda \rho_{b} w_{1} \lambda_{b}$ in Case 2 in Theorem 8.

If $w_{2}$ is empty then $v$ has prefix $z \lambda \rho w_{1} \lambda_{b} \mu_{b}$ with $w_{1} \in \mathcal{L}_{1, \infty}$ which is forbidden, so $w_{2}$ must move some tokens. The subword $w_{2}$ cannot leave any tokens in stack $B$. Either $w_{2}$ leaves some tokens in stack $A$, or not.

If $w_{2}$ leaves a token in stack $A$, this token cannot be one of $t_{r+1}, \ldots, t_{s}$ or else $v$ would generate a different permutation to $u$. Therefore this token is moved into stack $A$ after 
$t_{r}$ by a letter $\rho$. This letter cannot be followed by $\mu$, and since it remains in stack $A$ it is not followed by $\lambda$. So this letter is either the last letter of $w_{2}$, or is followed by another $\rho$, which must also remain in stack $A$. Thus $w_{2}$ ends with $\rho$, but this is a contradiction since $w_{2}$ is followed by $\mu_{b}$.

Thus $w_{2}$ does not leave any tokens in stacks $A$ or $B$, so moves $t_{r+1}, \ldots, t_{s}$ from the input to the output, and $w_{2} \in \mathcal{L}_{2, \infty}$. Note that $w_{1} w_{2}$ produces the same permutation of $t_{1}, \ldots, t_{s}$ as $w$ does, and $w \in \mathcal{L}_{1, \infty}$ so generates a 312 -avoiding permutation of $t_{1}, \ldots, t_{s}$. The subword $w_{1}$ permutes the first $r$ tokens, and so $w_{2}$ must produce a permutation of $t_{r+1}, \ldots, t_{s}$ that avoids 312 . In this case $v$ has prefix $z \lambda \rho_{b} w_{1} \lambda_{b} w_{2} \mu_{b}$ where $D_{\rho, \lambda}\left(z \lambda \rho_{b}\right)=1$, $w_{1} \in \mathcal{L}_{1, \infty}$ and $w_{2}$ generates a 312 -avoider, so by Lemma $7 v$ must also contain a prefix that is not allowed if $v \in \mathcal{L}$. This is a contradiction, so we cannot have two such words $u$ and $v$.

\subsection{A related class of permutations}

A natural question to ask is whether switching the order of the stacks makes any difference to the problem. Let $\mathcal{Q}$ be the set of permutations that can be generated by passing an ordered sequence through an infinite stack followed by a depth 2 stack in series. Each word $w \in \mathcal{L}_{2, \infty}$ encodes a permutation in $\mathcal{Q}$ as follows: reading $w$ from right to left, for each $\mu$ move a token from the input to the infinite stack, for each $\lambda$ move a token from the infinite stack to the depth 2 stack, and for each $\rho$ move a token from the depth 2 stack to the output. It follows that $\mathcal{P}$ and $\mathcal{Q}$ are in bijection.

\section{Constructing a pushdown automaton}

In this section we construct a deterministic pushdown automaton accepting on empty stack, which accepts the language

$$
\mathcal{L} \$=\{w \$ \mid w \in \mathcal{L}\}
$$

A pushdown automaton accepting on empty stack $M$ is the following:

1. $Q$ a finite set of states,

2. $\Sigma$ a finite input alphabet,

3. $\Gamma$ a finite stack alphabet,

4. $q_{0} \in Q$ the start state,

5. $0 \in \Gamma$ a special stack symbol,

6. a map $\delta$ from $Q \times(\Sigma \cup \varepsilon) \times \Gamma$ to finite subsets of $Q \times\left(\Gamma^{*}\right)$, called the transition function, 
which runs as follows. Before reading input, the stack contains a single 0. Input strings are accepted as soon as the stack becomes empty. A configuration of $M$ is a pair $(q, \omega)$ where $q$ is the current state and $\omega \in \Gamma^{*}$ is a string of stack symbols representing the contents of the stack (the first letter of $\omega$ is the top of the stack). The notation $\delta\left(q_{i}, a, k\right)=$ $\left\{\left(q_{j_{1}}, \gamma_{1}\right), \ldots,\left(q_{j_{s}}, \gamma_{s}\right)\right\}$ means that if $M$ has the configuration $\left(q_{i}, k \omega\right)$ and $a \in \Sigma \cup\{\varepsilon\}$ is the next input letter to be read, then $M$ can move to the configuration $\left(q_{j_{l}}, \gamma_{l} \omega\right)$ for some $1 \leqslant l \leqslant s$, removing the token $k$ from the top of the stack and replacing it by $\gamma_{l}$.

See [14] for more details.

A pushdown automaton is deterministic if for each state $q$ and stack symbol $i$

1. if $|\delta(q, \varepsilon, i)|=1$ then $|\delta(q, a, i)|=0$ for all $a \in \Sigma$,

2. for each $a \in \Sigma \cup\{\varepsilon\}$ the set $\delta(q, a, i)$ has size at most one.

Note that a determistic pushdown automaton accepting on empty stack cannot accept the empty string (unless this is the only string it accepts) since there would have to be a transition $\delta\left(q_{0}, \varepsilon, 0\right)$ as well as a transition $\delta\left(q_{0}, a, 0\right)$ for some letter $a$.

Let $M$ be the pushdown automaton shown in Figure 8, which accepts on empty stack. $M$ uses its stack to keep track of $D_{\lambda, \mu}$ as it reads its input, and its states to keep track of $D_{\rho, \lambda}$. It uses the stack symbol 2 as a device to flag when the input has the potential to have a prefix of the form $w_{0} \rho \lambda$ or $w_{0} \lambda \rho$ with $D_{\rho, \lambda}\left(w_{0}\right)=1$. Paths $\rho \mu$ are forbidden. We will prove that the language of this automaton is precisely the language $\mathcal{L} \$$.

Here is the formal description of $M$. Note that states $q_{3}, q_{6}, q_{7}$ are reached only when 1 is on top of the stack, and $q_{5}, q_{8}$ are reached when either 1 or 2 are on top of the stack, so we have omitted transitions from configurations that are not possible.

1. states $Q=\left\{q_{0}, \ldots, q_{8}\right\}$,

2. input alphabet $\Sigma=\{\rho, \lambda, \mu, \$\}$,

3. stack alphabet $\Gamma=\{0,1,2\}$,

4. start state $q_{0}$,

5. transition function $\delta$ defined as follows.

$\begin{array}{lll}\delta\left(q_{0}, \$, 0\right)=\left(q_{0}, \varepsilon\right) & \delta\left(q_{1}, \rho, 1\right)=\left(q_{2}, 1\right) & \delta\left(q_{1}, \lambda, 2\right)=\left(q_{3}, 12\right) \\ \delta\left(q_{0}, \mu, 1\right)=\left(q_{0}, \varepsilon\right) & \delta\left(q_{1}, \rho, 2\right)=\left(q_{2}, 2\right) & \delta\left(q_{2}, \lambda, 0\right)=\left(q_{5}, 10\right) \\ \delta\left(q_{0}, \mu, 2\right)=\left(q_{0}, \varepsilon\right) & \delta\left(q_{3}, \rho, 1\right)=\left(q_{6}, 1\right) & \delta\left(q_{2}, \lambda, 1\right)=\left(q_{5}, 11\right) \\ \delta\left(q_{3}, \mu, 1\right)=\left(q_{0}, \varepsilon\right) & \delta\left(q_{4}, \rho, 0\right)=\left(q_{2}, 0\right) & \delta\left(q_{2}, \lambda, 2\right)=\left(q_{5}, 12\right) \\ \delta\left(q_{4}, \mu, 1\right)=\left(q_{4}, \varepsilon\right) & \delta\left(q_{4}, \rho, 1\right)=\left(q_{2}, 1\right) & \delta\left(q_{4}, \lambda, 0\right)=\left(q_{3}, 10\right) \\ \delta\left(q_{4}, \mu, 2\right)=\left(q_{4}, \varepsilon\right) & \delta\left(q_{4}, \rho, 2\right)=\left(q_{2}, 2\right) & \delta\left(q_{4}, \lambda, 1\right)=\left(q_{3}, 11\right) \\ \delta\left(q_{5}, \mu, 1\right)=\left(q_{4}, \varepsilon\right) & \delta\left(q_{5}, \rho, 1\right)=\left(q_{8}, 1\right) & \delta\left(q_{4}, \lambda, 2\right)=\left(q_{3}, 12\right) \\ \delta\left(q_{5}, \mu, 2\right)=\left(q_{5}, \varepsilon\right) & \delta\left(q_{5}, \rho, 2\right)=\left(q_{8}, 2\right) & \delta\left(q_{5}, \lambda, 1\right)=\left(q_{7}, 11\right) \\ \delta\left(q_{0}, \rho, 0\right)=\left(q_{1}, 0\right) & \delta\left(q_{6}, \rho, 1\right)=\left(q_{8}, 1\right) & \delta\left(q_{5}, \lambda, 2\right)=\left(q_{7}, 12\right) \\ \delta\left(q_{0}, \rho, 1\right)=\left(q_{1}, 1\right) & \delta\left(q_{7}, \rho, 1\right)=\left(q_{6}, 1\right) & \delta\left(q_{6}, \lambda, 1\right)=\left(q_{7}, 11\right) \\ \delta\left(q_{0}, \rho, 2\right)=\left(q_{1}, 2\right) & \delta\left(q_{1}, \lambda, 0\right)=\left(q_{3}, 10\right) & \delta\left(q_{8}, \lambda, 1\right)=\left(q_{5}, 21\right) \\ \delta\left(q_{1}, \rho, 0\right)=\left(q_{2}, 0\right) & \delta\left(q_{1}, \lambda, 1\right)=\left(q_{3}, 11\right) & \delta\left(q_{8}, \lambda, 2\right)=\left(q_{5}, 22\right)\end{array}$




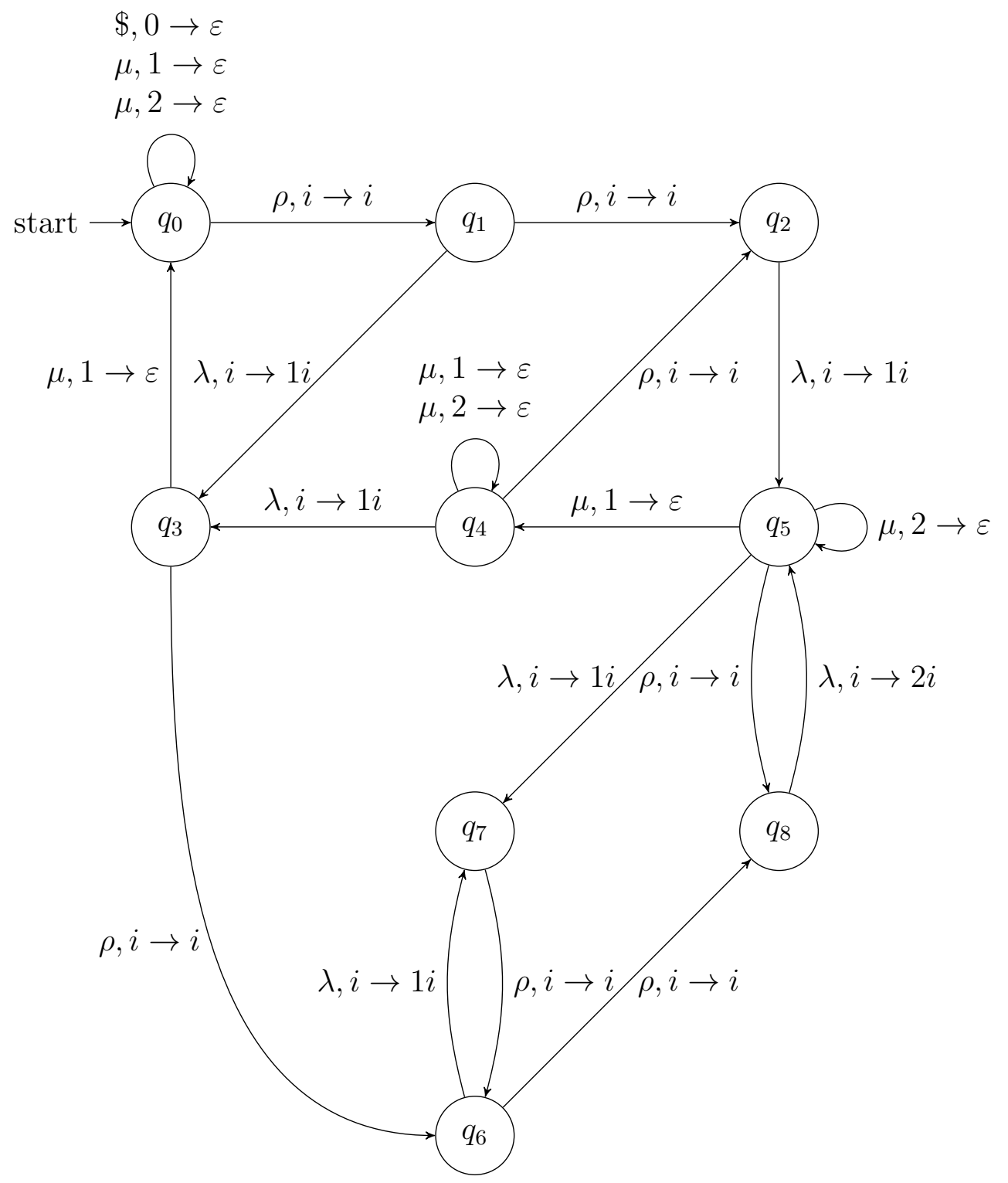

Figure 8: Pushdown automaton $M$ accepting on empty stack, with start configuration $\left(q_{0}, 0\right)$. The symbol $i \in\{0,1,2\}$ represents a stack token that is kept in place by a transition.

To prove that $M$ accepts precisely the language $\mathcal{L} \$$, we first show that $M$ is deterministic. This allows us to identify input words with unique paths in $M$ and simplify our arguments slightly.

Lemma 9. The pushdown automaton $M$ is deterministic.

Proof. The claim is easily verified by considering the formal description for $M$.

Proposition 10. The pushdown automaton $M$ accepts the language $\mathcal{L} \$=\{w \$ \mid w \in \mathcal{L}\}$. 
Proof. Since $M$ is deterministic, we identify input words with their corresponding unique path in $M$.

Let $w \in\{\rho, \lambda, \mu\}^{*}$. We must show that

1. if $w$ contains $\rho \mu$, then $w \$$ is rejected.

2. if $w$ fails to be in $\mathcal{L}_{2, \infty}$, then $w \$$ is rejected,

3. if $w$ has a bad prefix (conditions (2) and (3) in Definition 6), then $w \$$ rejected.

4. if $w \$$ is rejected, then $w \notin \mathcal{L}$.

The only states that can be reached by a path $u \rho$ for $u \in\{\rho, \lambda, \mu\}^{*}$ from the start configuration are $q_{1}, q_{2}, q_{6}$ and $q_{8}$ and since none are the source of a $\mu$ transition, any word containing $\rho \mu$ will be rejected.

Next, we show that if $w$ is not in $\mathcal{L}_{2, \infty}$, then $w \$$ is rejected by $M$. Each state represents the endpoint of a path labeling a prefix of an input string accepted by the automaton. One can verify the values of $D_{\rho, \lambda}(u)$ for each path labeled $u$ ending at state $q_{i}$ given by Table 1.

\begin{tabular}{|c||c|c|c|c|c|c|c|c|c|}
\hline state & $q_{0}$ & $q_{1}$ & $q_{2}$ & $q_{3}$ & $q_{4}$ & $q_{5}$ & $q_{6}$ & $q_{7}$ & $q_{8}$ \\
\hline$D_{\rho, \lambda}$ & 0 & 1 & 2 & 0 & 1 & 1 & 1 & 0 & 2 \\
\hline
\end{tabular}

Table 1: Value of $D_{\rho, \lambda}$ for any prefix ending at each state

Let $h(u)$ be the height of the second (infinite) stack after reading $u \in\{\rho, \lambda, \mu\}^{*}$ starting from the start configuration $\left(q_{0}, 0\right)$. Then $h(\varepsilon)=1, h(u \rho)=h(u), h(u \lambda)=h(u)+1$ and $h(u \mu)=h(u)-1$ since $\lambda$ pushes a token to the stack, $\mu$ pops a token and $\rho$ keeps the stack unchanged. It follows that $h(u)=D_{\lambda, \mu}(u)+1$, and since 0 stays on the stack until $\$$ is read, $h(u) \geqslant 1$ for all prefixes $u \in\{\rho, \lambda, \mu\}^{*}$, so $D_{\lambda, \mu}(u) \geqslant 0$. If $w \$$ is accepted then the stack must contain only 0 after reading $w$, so $D_{\lambda, \mu}(w)=0$.

It follows that if $D_{\rho, \lambda}(u)>2, D_{\rho, \lambda}(u), D_{\lambda, \mu}(u)<0$ for some prefix $u$, or $D_{\lambda, \mu}(w) \neq 0$, then $M$ will reject $w \$$.

Next, suppose $w \in \mathcal{L}_{2, \infty}$ has no $\rho \mu$ substring and a prefix of the form $w_{0} v w_{1} \lambda \mu$ where $D_{\rho, \lambda}\left(w_{0}\right)=1, v \in\{\rho \lambda, \lambda \rho\}$ and $w_{1} \in \mathcal{L}_{1, \infty}$. The string $w_{0}$ labels a path in the automaton starting at $q_{0}$ and ending at state $q_{1}, q_{4}, q_{5}$ or $q_{6}$ by Table 1. From each of these states, reading $v=\rho \lambda$ ends in state $q_{5}$, and reading $v=\lambda \rho$ ends in state $q_{6}$.

From $q_{5}$, the word $w_{1}$ labels a path that visits only states $q_{5}$ and $q_{8}$, since $D_{\lambda, \mu}(z) \geqslant 0$ for all prefixes $z$ of $w_{1}$, so the 1 on top of the stack before reading $w_{1}$ remains (and is covered by $2 \mathrm{~s}$, which are removed by the $\mu$ loop at $\left.q_{5}\right)$, and ends at $q_{5}$ since $D_{\lambda, \mu}\left(w_{1}\right)=0$. From here reading $\lambda \mu$ is rejected.

From $q_{6}$, if $w_{1}=\varepsilon$ then $u \lambda \rho \lambda \mu$ is rejected. Otherwise $w_{1}$ labels a path from $q_{6}$ to $q_{8}$ and then moves between $q_{5}$ and $q_{8}$, and ends at $q_{5}$. From here reading $\lambda \mu$ is rejected.

We have now established that if $w \notin \mathcal{L}$ then $w \$$ is rejected by $M$. To complete the proof we must show that if $w \$$ is rejected, then $w \notin \mathcal{L}$. To show this, assume $w \in \mathcal{L}_{2, \infty}$ 
with no $\rho \mu$ substring, but $w \$$ is rejected by $M$. We will prove that $w$ must have a bad prefix.

Let $p$ be the longest prefix of $w \$$ labeling a path that is not rejected by $M$. Since $w \in \mathcal{L}_{2, \infty}$ we have $D_{\lambda, \mu}(w)=0$, so if $w=p$, after reading $w$ the stack contains just 0 so $w \$$ will be accepted, a contradiction. Thus $p$ is strictly shorter than $w$. Let $w=p x w^{\prime}$ where $x \in\{\rho \lambda, \mu\}$ is the next letter input after reading $p$.

We now consider the possible states where $p$ can end.

1. Suppose $p$ ends at $q_{0}$. Then $D_{\rho, \lambda}(p)=0$ so $x \neq \lambda$. If the top of stack is 0 then $D_{\lambda, \mu}(p)=0$ so $x \neq \mu$. Otherwise $M$ cannot reject on reading $\rho, \mu$.

2. Suppose $p$ ends at $q_{1}$, so its last letter is $\rho$, and $D_{\rho, \lambda}(p)=1$. Then $x \neq \mu$. Otherwise $M$ cannot reject on reading $\rho, \lambda$.

3. Suppose $p$ ends at $q_{2}$, so its last letter is $\rho$, and $D_{\rho, \lambda}(p)=2$. Then $x \neq \mu, \rho$. Otherwise $M$ cannot reject on reading $\lambda$.

4. Suppose $p$ ends at $q_{3}$, so $D_{\rho, \lambda}(p)=0$ and the top of stack is 1 . Then $x \neq \lambda$. Otherwise $M$ cannot reject on reading $\rho,(\mu, 1 \rightarrow \varepsilon)$.

5. Suppose $p$ ends at $q_{4}$, so $D_{\rho, \lambda}(p)=1$. The only way $M$ could reject is if the top of stack is 0 and $x=\mu$, which is not possible since $w \in \mathcal{L}_{2, \infty}$.

6. Suppose $p$ ends at $q_{5}$, so $D_{\rho, \lambda}(p)=1$ and 1 is on top of the stack. Then no letter will cause $M$ to reject.

7. Suppose $p$ ends at $q_{6}$, so $D_{\rho, \lambda}(p)=1$ and $p$ ends with $\lambda \rho$. Then $x$ cannot be $\mu$, and otherwise $p x$ is not rejected.

8. Suppose $p$ ends at $q_{8}$, so its last letter is $\rho$, and $D_{\rho, \lambda}(p)=2$. Then $x \neq \mu, \rho$ and $M$ cannot reject if $x=\lambda$.

These cases show that if $p$ ends at any state except $q_{7}$, then $M$ does not reject $w$ on reading the next input letter. We finish the proof by showing that if $p$ ends at $q_{7}$, then $p x$ is a bad prefix.

Since $p$ ends at $q_{7}, p$ ends with $\lambda, D_{\rho, \lambda}(p)=2$, and $D_{\lambda, \mu}(p)>0$. If $x=\rho$ then $p x$ is not rejected. If $x=\lambda$ then $w \notin \mathcal{L}_{2, \infty}$. So we must have $x=\mu$.

Let $p=p_{1} \lambda$. If $p_{1}$ ends at $q_{6}$, then $p_{1}=p_{2} \lambda \rho$, and $p x=p_{2} \lambda \rho \lambda \mu$ where $D_{\rho, \lambda}\left(p_{2}\right)=1$ and so $p x$ is a bad prefix. The machine correctly rejects the string on reading $x=\mu$.

Otherwise $p_{1}$ ends at $q_{5}$. Either $p_{1}$ ends with $\rho \lambda$, or $\mu$. If $p_{1}=p_{2} \rho \lambda$ then $D_{\rho, \lambda}\left(p_{2}\right)=1$ and $p x=p_{2} \rho \lambda \lambda \mu$ is a bad prefix. Otherwise $p_{1}$ ends in $\mu$, and must pop a token 2 from the stack. Let $\lambda_{*}$ be the last $\lambda$ letter in $p_{1}$ that pushed a 1 on top of the stack (which must exist, since all paths to $q_{5}$ must cross such an edge). Write $p_{1}=p_{2} \lambda_{*} p_{3} \mu$. 
The letter $\lambda_{*}$ labels one of the following edges:

1. from $q_{2}$ to $q_{5}$,

2. from $q_{1}$ to $q_{3}$,

3. from $q_{4}$ to $q_{3}$,

4. from $q_{5}$ to $q_{7}$,

5. from $q_{6}$ to $q_{7}$.

In the first case, $p_{2}$ ends at $q_{2}$ so must have the form $p_{2}=u \rho$ with $D_{\rho, \lambda}(u)=1$. Then $p_{3} \mu$ labels a path that moves between states $q_{5}$ and $q_{8}$, reading $\rho \lambda$ and pushing a 2 , or reading $\mu$ and popping a 2 , so $p_{3} \mu \in \mathcal{L}_{1, \infty}$. It follows that $w$ has the bad prefix $u \rho \lambda_{*}\left(p_{3} \mu\right) \lambda \mu$, and so $M$ correctly rejects it.

In the other four cases we have that $D_{\rho, \lambda}\left(p_{2}\right)=1$ since $p_{2}$ ends at state $q_{1}, q_{4}, q_{5}$ or $q_{6}$, $\lambda_{*}$ must be immediately followed by a letter $\rho$, and $p_{2} \lambda_{*} \rho$ ends at state $q_{6}$. Let $p_{3}=\rho p_{4}$. Then $p_{4} \mu$ labels a path that starts at $q_{6}$, goes to $q_{8}$, then moves between states $q_{5}$ and $q_{8}$, reading $\rho \lambda$ and pushing a 2 , or reading $\mu$ and popping a 2 . So $p_{4} \mu \in \mathcal{L}_{1, \infty}$. It follows that $w$ has the bad prefix $p_{2} \lambda_{*} \rho\left(p_{4} \mu\right) \lambda \mu$, and so $M$ correctly rejects it.

\section{Obtaining the generating function}

Theorem 11. The sequence counting the number of permutations of each length in $\mathcal{P}$ has an algebraic generating function:

$$
\sum_{n \geqslant 0} c_{n} z^{n}=\frac{(1+q)\left(1+5 q-q^{2}-q^{3}-(1-q) \sqrt{\left(1-q^{2}\right)\left(1-4 q-q^{2}\right)}\right)}{8 q}
$$

where $c_{n}$ is the number of permutations in $\mathcal{P}$ of length $n$, and $q \equiv q(z)=\frac{1-2 z-\sqrt{1-4 z}}{2 z}$.

Proof. We convert the pushdown automaton given in the previous section to an unambiguous context-free language, following the standard procedure as described in Hopcroft and Ullman [14]. Theorem 10.12 of Hopcroft and Ullman guarantees that the grammar obtained from a deterministic pushdown automaton accepting on empty stack is $L R(0)$ and hence unambiguous.

We then apply the Chomsky and Schützenberger theorem, as outlined for example in [12] I.5.4, to obtain an algebraic generating function. Since each step in this procedure is constructive, we can find the generating function explicitly.

We start by converting the pushdown automaton to a grammar. See Theorem 5.4 [14] for full details.

Define a grammar with nonterminals $S$ and $\left[q_{i}, j, q_{k}\right]=N_{i, j, k}$ for each pair of states $q_{i}, q_{k}$ and stack symbol $j$. The nonterminal $\left[q_{i}, j, q_{k}\right]$ represents a path in the configuration space of the pushdown automaton starting at $q_{i}$ with $j$ on top of the stack and ending 
at some state $q_{k}$. The productions "fill out" these paths with subpaths according to the transitions that are possible.

The production rules are then defined as follows:

1. for each state $q_{i}$ we have a production $S \rightarrow N_{00 i}$,

2. for each transition $\delta\left(q_{i}, a, j\right)=\left\{\left(q_{k}, \varepsilon\right)\right\}$ with $a \in\{\$, \mu\}$, add a production $N_{i j k}=a$,

3. for each transition $\delta\left(q_{i}, \rho, j\right)=\left\{\left(q_{k}, l\right)\right\}$, add productions $N_{i j x}=\rho N_{k l x}$ for $0 \leqslant x \leqslant 8$,

4. for each transition $\delta\left(q_{i}, \lambda, j\right)=\left\{\left(q_{k}, l m\right)\right\}$, add productions $N_{i j x}=\lambda N_{k l y} N_{y m x} 0 \leqslant$ $x, y \leqslant 8$.

This gives the following set of productions, where $0 \leqslant x, y \leqslant 8$ :

\begin{tabular}{|c|c|c|c|c|c|c|}
\hline$N_{000}$ & $\rightarrow \$$ & $N_{41 x}$ & $\rightarrow$ & $\rho N_{21 x}$ & $N_{12 x} \rightarrow$ & $\lambda N_{31 y} N_{y 2 x}$ \\
\hline$N_{010}$ & $\rightarrow \mu$ & $N_{42 x}$ & $\rightarrow$ & $\rho N_{22 x}$ & $N_{20 x} \rightarrow$ & $\lambda N_{51 y} N_{y 0 x}$ \\
\hline$N_{020}$ & $\rightarrow \mu$ & $N_{11 x}$ & $\rightarrow$ & $\rho N_{21 x}$ & $N_{21 x} \rightarrow$ & $\lambda N_{51 y} N_{y 1 x}$ \\
\hline$N_{310}$ & $\rightarrow \mu$ & $N_{12 x}$ & $\rightarrow$ & $\rho N_{22 x}$ & $N_{22 x} \rightarrow$ & $\lambda N_{51 y} N_{y 2 x}$ \\
\hline$N_{414}$ & $\rightarrow \mu$ & $N_{31 x}$ & $\rightarrow$ & $\rho N_{61 x}$ & $N_{40 x} \rightarrow$ & $\lambda N_{31 y} N_{y 0 x}$ \\
\hline$N_{424}$ & $\rightarrow \mu$ & $N_{40 x}$ & $\rightarrow$ & $\rho N_{20 x}$ & $N_{41 x} \rightarrow$ & $\lambda N_{31 y} N_{y 1 x}$ \\
\hline$N_{514}$ & $\rightarrow \mu$ & $N_{51 x}$ & $\rightarrow$ & $\rho N_{81 x}$ & $N_{42 x} \rightarrow$ & $\lambda N_{31 y} N_{y 2 x}$ \\
\hline$\rightarrow$ & $\mu$ & $N_{52 x}$ & $\rightarrow$ & $\rho N_{82 x}$ & $N_{51 x}$ & $\rightarrow \quad \lambda N_{71 y} N_{y 1 x}$ \\
\hline$\rightarrow$ & $\rho N_{10 x}$ & $N_{61 x}$ & $\rightarrow$ & $\rho N_{81 x}$ & $N_{52 x}$ & $\rightarrow \quad \lambda N_{71 y} N_{y 2 x}$ \\
\hline$\rightarrow$ & $\rho N_{11 x}$ & $N_{71 x}$ & $\rightarrow$ & $\rho N_{61 x}$ & $N_{61 x}$ & $\rightarrow \quad \lambda N_{71 y} N_{y 1 x}$ \\
\hline$\rightarrow$ & $\rho N_{12 x}$ & $N_{10 x}$ & $\rightarrow$ & $\lambda N_{31 y} N_{y 0 x}$ & $N_{81 x}$ & $\rightarrow \quad \lambda N_{52 y} N_{y 1 x}$ \\
\hline$\rightarrow$ & $\rho N_{20 x}$ & $N_{11 x}$ & $\rightarrow$ & $\lambda N_{31 y} N_{y 1 x}$ & $N_{82 x}$ & $\rightarrow \quad \lambda N_{52 y} N_{y 2 x}$ \\
\hline
\end{tabular}

We can reduce the size of the grammar description as follows. First, observe that the only productions that eliminate nonterminals (by generating $\$$ or $\mu$ ) are of the form $N_{* j k}$ for $k \in\{0,4,5\}$, and $j=0$ implies $k=0$. Since all productions with nonterminals on the right side have the form $N_{* i j} \rightarrow \rho N_{* i j}$ or $N_{* i j} \rightarrow \lambda N_{* * *} N_{* i j}$, it follows that any nonterminal $N_{* * k}$ with $k$ not equal to 0,4 or 5 cannot be eliminated, so we can exclude them from the grammar.

Also, if we start a derivation with $S \rightarrow N_{00 k}$ for $k \neq 0$, there will always be a nonterminal of the form $N_{* 0 k}$ that cannot be eliminated. Therefore it suffices to make $N_{000}$ the start nonterminal and remove all productions involving $S$.

Lastly, the resulting grammar contain nonterminals $N_{500}, N_{504}, N_{505}$ that will never produce a string of only terminals, since the configuration $\left(q_{5}, 0\right)$ is never realised (to reach $q_{5}$ the top of stack symbol is either 1 or 2 . We modify the above grammar one step further by removing any production involving these nonterminals.

Taking these factors into consideration, and collecting productions with the same left side together we obtain the following grammar: 


$$
\begin{aligned}
& N_{000} \rightarrow \$ \mid \rho N_{100}, \\
& N_{004} \rightarrow \rho N_{104} \\
& N_{005} \rightarrow \rho N_{105} \\
& N_{010} \rightarrow \mu \mid \rho N_{110}, \\
& N_{014} \rightarrow \rho N_{114} \\
& N_{015} \rightarrow \rho N_{115} \\
& N_{020} \rightarrow \mu \mid \rho N_{120}, \\
& N_{024} \rightarrow \rho N_{124} \\
& N_{025} \rightarrow \rho N_{125} \\
& \begin{array}{ll|l|l}
N_{100} \rightarrow \rho N_{200} & \lambda N_{310} N_{000} & \lambda N_{314} N_{400},
\end{array} \\
& \begin{array}{lll|l|l}
N_{104} \rightarrow \rho N_{204} & \lambda N_{310} N_{004} & \lambda N_{314} N_{404},
\end{array}
\end{aligned}
$$

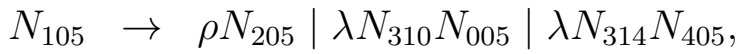

$$
\begin{aligned}
& N_{110} \rightarrow \rho N_{210}\left|\lambda N_{310} N_{010}\right| \lambda N_{314} N_{410} \mid \lambda N_{315} N_{510}, \\
& \begin{array}{ll|l|l|l}
N_{114} \rightarrow \rho N_{214} & \lambda N_{310} N_{014} & \lambda N_{314} N_{414} & \lambda N_{315} N_{514},
\end{array} \\
& \begin{array}{ll|l|l|l}
N_{115} \rightarrow \rho N_{215} & \lambda N_{310} N_{015} & \lambda N_{314} N_{415} & \lambda N_{315} N_{515},
\end{array} \\
& N_{120} \rightarrow \rho N_{220}\left|\lambda N_{310} N_{020}\right| \lambda N_{314} N_{420} \mid \lambda N_{315} N_{520}, \\
& N_{124} \rightarrow \rho N_{224}\left|\lambda N_{310} N_{024}\right| \lambda N_{314} N_{424} \mid \lambda N_{315} N_{524},
\end{aligned}
$$

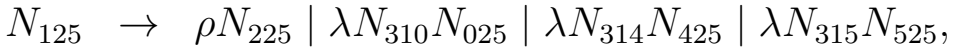

$$
\begin{aligned}
& N_{200} \rightarrow \lambda N_{510} N_{000} \mid \lambda N_{514} N_{400}
\end{aligned}
$$

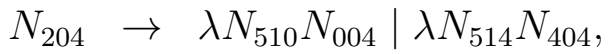

$$
\begin{aligned}
& N_{205} \rightarrow \lambda N_{510} N_{005} \mid \lambda N_{514} N_{405},
\end{aligned}
$$

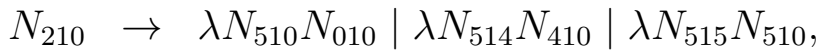

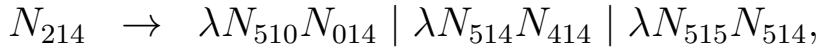

$$
\begin{aligned}
& N_{215} \rightarrow \lambda N_{510} N_{015}\left|\lambda N_{514} N_{415}\right| \lambda N_{515} N_{515}, \\
& N_{220} \rightarrow \lambda N_{510} N_{020}\left|\lambda N_{514} N_{420}\right| \lambda N_{515} N_{520},
\end{aligned}
$$

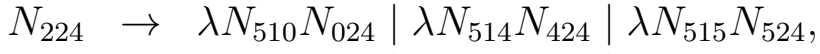

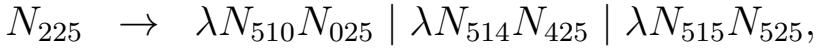

$$
\begin{aligned}
& N_{310} \rightarrow \mu \mid \rho N_{610}, \\
& N_{314} \rightarrow \rho N_{614} \\
& N_{315} \rightarrow \rho N_{615}
\end{aligned}
$$

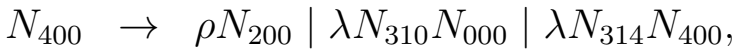

$$
\begin{aligned}
& \begin{array}{ll|l|l}
N_{404} \rightarrow \rho N_{204} & \lambda N_{310} N_{004} & \lambda N_{314} N_{404},
\end{array} \\
& \begin{array}{ll|l|l}
N_{405} \rightarrow \rho N_{205} & \lambda N_{310} N_{005} & \lambda N_{314} N_{405},
\end{array} \\
& N_{410} \rightarrow \rho N_{210}\left|\lambda N_{310} N_{010}\right| \lambda N_{314} N_{410} \mid \lambda N_{315} N_{510} \text {, } \\
& N_{414} \rightarrow \mu\left|\rho N_{214}\right| \lambda N_{310} N_{014}\left|\lambda N_{314} N_{414}\right| \lambda N_{315} N_{514} \text {, }
\end{aligned}
$$

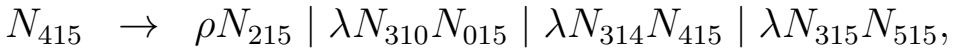

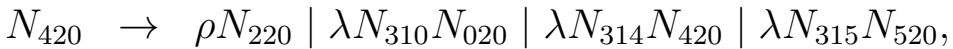

$$
\begin{aligned}
& N_{424} \rightarrow \mu\left|\rho N_{224}\right| \lambda N_{310} N_{024}\left|\lambda N_{314} N_{424}\right| \lambda N_{315} N_{524} \text {, } \\
& N_{425} \rightarrow \rho N_{225}\left|\lambda N_{310} N_{025}\right| \lambda N_{314} N_{425} \mid \lambda N_{315} N_{525} \text {, }
\end{aligned}
$$




$$
\begin{aligned}
& N_{510} \rightarrow \rho N_{810}\left|\lambda N_{710} N_{010}\right| \lambda N_{714} N_{410} \mid \lambda N_{715} N_{510}, \\
& N_{514} \rightarrow \mu\left|\rho N_{814}\right| \lambda N_{710} N_{014}\left|\lambda N_{714} N_{414}\right| \lambda N_{715} N_{514}, \\
& N_{515} \rightarrow \rho N_{815}\left|\lambda N_{710} N_{015}\right| \lambda N_{714} N_{415} \mid \lambda N_{715} N_{515}, \\
& N_{520} \rightarrow \rho N_{820}\left|\lambda N_{710} N_{020}\right| \lambda N_{714} N_{420} \mid \lambda N_{715} N_{520}, \\
& N_{524} \rightarrow \rho N_{824}\left|\lambda N_{710} N_{024}\right| \lambda N_{714} N_{424} \mid \lambda N_{715} N_{524}, \\
& N_{525} \rightarrow \mu\left|\rho N_{825}\right| \lambda N_{710} N_{025}\left|\lambda N_{714} N_{425}\right| \lambda N_{715} N_{525}, \\
& N_{610} \rightarrow \rho N_{810}\left|\lambda N_{710} N_{010}\right| \lambda N_{714} N_{410} \mid \lambda N_{715} N_{510}, \\
& N_{614} \rightarrow \rho N_{814}\left|\lambda N_{710} N_{014}\right| \lambda N_{714} N_{414} \mid \lambda N_{715} N_{514}, \\
& N_{615} \rightarrow \rho N_{815}\left|\lambda N_{710} N_{015}\right| \lambda N_{714} N_{415} \mid \lambda N_{715} N_{515}, \\
& N_{710} \rightarrow \rho N_{610}, \\
& N_{714} \rightarrow \rho N_{614}, \\
& N_{715} \rightarrow \rho N_{615}, \\
& N_{810} \rightarrow \lambda N_{520} N_{010}\left|\lambda N_{524} N_{410}\right| \lambda N_{525} N_{510}, \\
& N_{814} \rightarrow \lambda N_{520} N_{014}\left|\lambda N_{524} N_{414}\right| \lambda N_{525} N_{514}, \\
& N_{815} \rightarrow \lambda N_{520} N_{015}\left|\lambda N_{524} N_{415}\right| \lambda N_{525} N_{515}, \\
& N_{820} \rightarrow \lambda N_{520} N_{020}\left|\lambda N_{524} N_{420}\right| \lambda N_{525} N_{520}, \\
& N_{824} \rightarrow \lambda N_{520} N_{024}\left|\lambda N_{524} N_{424}\right| \lambda N_{525} N_{524}, \\
& N_{825} \rightarrow \lambda N_{520} N_{025}\left|\lambda N_{524} N_{425}\right| \lambda N_{525} N_{525} .
\end{aligned}
$$

The next step is to convert nonterminals to generating functions, terminals to $z$ and productions to equations, as described in [12] I.5.4.

$$
\begin{aligned}
& f_{000}=z+z f_{100}, \\
& f_{004}=z f_{104}, \\
& f_{005}=z f_{105}, \\
& f_{010}=z+z f_{110}, \\
& f_{014}=z f_{114}, \\
& f_{015}=z f_{115}, \\
& f_{020}=z+z f_{120}, \\
& f_{024}=z f_{124}, \\
& f_{025}=z f_{125}, \\
& f_{100}=z f_{200}+z f_{310} f_{000}+z f_{314} f_{400}, \\
& f_{104}=z f_{204}+z f_{310} f_{004}+z f_{314} f_{404}, \\
& f_{105}=z f_{205}+z f_{310} f_{005}+z f_{314} f_{405}, \\
& f_{110}=z f_{210}+z f_{310} f_{010}+z f_{314} f_{410}+z f_{315} f_{510}, \\
& f_{114}=z f_{214}+z f_{310} f_{014}+z f_{314} f_{414}+z f_{315} f_{514}, \\
& f_{115}=z f_{215}+z f_{310} f_{015}+z f_{314} f_{415}+z f_{315} f_{515}, \\
& f_{120}=z f_{220}+z f_{310} f_{020}+z f_{314} f_{420}+z f_{315} f_{520}, \\
& f_{124}=z f_{224}+z f_{310} f_{024}+z f_{314} f_{424}+z f_{315} f_{524}, \\
& f_{125}=z f_{225}+z f_{310} f_{025}+z f_{314} f_{425}+z f_{315} f_{525},
\end{aligned}
$$




$$
\begin{aligned}
& f_{200}=z f_{510} f_{000}+z f_{514} f_{400}, \\
& f_{204}=z f_{510} f_{004}+z f_{514} f_{404} \text {, } \\
& f_{205}=z f_{510} f_{005}+z f_{514} f_{405} \text {, } \\
& f_{210}=z f_{510} f_{010}+z f_{514} f_{410}+z f_{515} f_{510}, \\
& f_{214}=z f_{510} f_{014}+z f_{514} f_{414}+z f_{515} f_{514}, \\
& f_{215}=z f_{510} f_{015}+z f_{514} f_{415}+z f_{515} f_{515} \text {, } \\
& f_{220}=z f_{510} f_{020}+z f_{514} f_{420}+z f_{515} f_{520}, \\
& f_{224}=z f_{510} f_{024}+z f_{514} f_{424}+z f_{515} f_{524}, \\
& f_{225}=z f_{510} f_{025}+z f_{514} f_{425}+z f_{515} f_{525}, \\
& f_{310}=z+z f_{610} \text {, } \\
& f_{314}=z f_{614}, \\
& f_{315}=z f_{615}, \\
& f_{400}=z f_{200}+z f_{310} f_{000}+z f_{314} f_{400}, \\
& f_{404}=z f_{204}+z f_{310} f_{004}+z f_{314} f_{404} \text {, } \\
& f_{405}=z f_{205}+z f_{310} f_{005}+z f_{314} f_{405} \text {, } \\
& f_{410}=z f_{210}+z f_{310} f_{010}+z f_{314} f_{410}+z f_{315} f_{510}, \\
& f_{414}=z+z f_{214}+z f_{310} f_{014}+z f_{314} f_{414}+z f_{315} f_{514} \text {, } \\
& f_{415}=z f_{215}+z f_{310} f_{015}+z f_{314} f_{415}+z f_{315} f_{515} \text {, } \\
& f_{420}=z f_{220}+z f_{310} f_{020}+z f_{314} f_{420}+z f_{315} f_{520}, \\
& f_{424}=z+z f_{224}+z f_{310} f_{024}+z f_{314} f_{424}+z f_{315} f_{524} \text {, } \\
& f_{425}=z f_{225}+z f_{310} f_{025}+z f_{314} f_{425}+z f_{315} f_{525}, \\
& f_{510}=z f_{810}+z f_{710} f_{010}+z f_{714} f_{410}+z f_{715} f_{510} \text {, } \\
& f_{514}=z+z f_{814}+z f_{710} f_{014}+z f_{714} f_{414}+z f_{715} f_{514} \text {, } \\
& f_{515}=z f_{815}+z f_{710} f_{015}+z f_{714} f_{415}+z f_{715} f_{515} \text {, } \\
& f_{520}=z f_{820}+z f_{710} f_{020}+z f_{714} f_{420}+z f_{715} f_{520} \text {, } \\
& f_{524}=z f_{824}+z f_{710} f_{024}+z f_{714} f_{424}+z f_{715} f_{524} \text {, } \\
& f_{525}=z+z f_{825}+z f_{710} f_{025}+z f_{714} f_{425}+z f_{715} f_{525} \text {, } \\
& f_{610}=z f_{810}+z f_{710} f_{010}+z f_{714} f_{410}+z f_{715} f_{510} \text {, } \\
& f_{614}=z f_{814}+z f_{710} f_{014}+z f_{714} f_{414}+z f_{715} f_{514} \text {, } \\
& f_{615}=z f_{815}+z f_{710} f_{015}+z f_{714} f_{415}+z f_{715} f_{515} \text {, } \\
& f_{710}=z f_{610}, \\
& f_{714}=z f_{614}, \\
& f_{715}=z f_{615}, \\
& f_{810}=z f_{520} f_{010}+z f_{524} f_{410}+z f_{525} f_{510}, \\
& f_{814}=z f_{520} f_{014}+z f_{524} f_{414}+z f_{525} f_{514}, \\
& f_{815}=z f_{520} f_{015}+z f_{524} f_{415}+z f_{525} f_{515} \text {, } \\
& f_{820}=z f_{520} f_{020}+z f_{524} f_{420}+z f_{525} f_{520}, \\
& f_{824}=z f_{520} f_{024}+z f_{524} f_{424}+z f_{525} f_{524} \text {, } \\
& f_{825}=z f_{520} f_{025}+z f_{524} f_{425}+z f_{525} f_{525} \text {. }
\end{aligned}
$$

Using Maple (version 14) we can solve to obtain an expression for the algebraic generating function $f_{000}(z)$, which counts the number of words in $\mathcal{L} \$$ of each length. Since words in $\mathcal{L} \$$ of length $3 n+1$ are in bijection with permutations in $\mathcal{P}$ of length $n$, the 
generating function $\sum_{n \geqslant 0} c_{n} t^{n}$ where $c_{n}$ is the number of permutations of length $n$ in $\mathcal{P}$ is obtained by dividing $f_{000}$ by $z$ and substituting $z^{3}=t$.

From the expression for the generating function we can easily obtain the first few terms of the sequence:

$1+z+2 z^{2}+6 z^{3}+24 z^{4}+114 z^{5}+592 z^{6}+3216 z^{7}+17904 z^{8}+101198 z^{9}+578208 z^{10}+$ $3332136 z^{11}+19343408 z^{12}+\ldots$

We can also use standard analytic combinatorial methods [12] to deduce the asymptotic growth of the number of such permutations:

$$
c_{n} \sim \frac{\sqrt{25-11 \sqrt{5}}}{2 \sqrt{\pi n^{3}}} \cdot(2+2 \sqrt{5})^{n} \cdot\left(1+O\left(n^{-1}\right)\right) .
$$

\section{Acknowledgements}

The bulk of this paper is the result of a University of Newcastle summer vacation project undertaken by the second author under supervision of the first. Research was supported by the Australian Research Council (ARC) grant FT110100178, and the Natural Sciences and Engineering Research Council of Canada (NSERC).

\section{References}

[1] M. H. Albert, M. D. Atkinson, and N. Ruškuc. Regular closed sets of permutations. Theoret. Comput. Sci., 306(1-3):85-100, 2003.

[2] M. H. Albert, M. Elder, A. Rechnitzer, P. Westcott, and M. Zabrocki. On the Stanley-Wilf limit of 4231-avoiding permutations and a conjecture of Arratia. Adv. in Appl. Math., 36(2):96-105, 2006.

[3] Michael Albert and Mireille Bousquet-Mélou. Permutations sortable by two stacks in parallel and quarter plane walks. Eur. J. Comb., 43:131-164, 2015.

[4] Michael H. Albert, M. D. Atkinson, and Vincent Vatter. Counting 1324, 4231avoiding permutations. Electron. J. Combin., 16(1):\#R136, 2009.

[5] Michael H. Albert, Steve Linton, and Nik Ruškuc. The insertion encoding of permutations. Electron. J. Combin., 12:\#R47, 2005.

[6] M. D. Atkinson, M. J. Livesey, and D. Tulley. Permutations generated by token passing in graphs. Theoret. Comput. Sci., 178(1-2):103-118, 1997.

[7] M. D. Atkinson, M. M. Murphy, and N. Ruškuc. Sorting with two ordered stacks in series. Theoret. Comput. Sci., 289(1):205-223, 2002.

[8] Miklós Bóna. Exact enumeration of 1342-avoiding permutations: a close link with labeled trees and planar maps. J. Combin. Theory Ser. A, 80(2):257-272, 1997.

[9] Andrew R. Conway and Anthony J. Guttmann. On 1324-avoiding permutations. Adv. in Appl. Math., 64:50-69, 2015. 
[10] M. Elder. Pattern avoiding permutations are context-sensitive, 2004. arXiv: math/0412019

[11] Murray Elder. Permutations generated by a stack of depth 2 and an infinite stack in series. Electron. J. Combin., 13(1):\#R68, 2006.

[12] Philippe Flajolet and Robert Sedgewick. Analytic combinatorics. Cambridge University Press, Cambridge, 2009.

[13] Ira M. Gessel. Symmetric functions and P-recursiveness. J. Combin. Theory Ser. A, 53(2):257-285, 1990.

[14] John E. Hopcroft and Jeffrey D. Ullman. Introduction to automata theory, languages, and computation. Addison-Wesley Publishing Co., Reading, Mass., 1979. AddisonWesley Series in Computer Science.

[15] Donald E. Knuth. The art of computer programming. Volume 3. Addison-Wesley Publishing Co., Reading, Mass.-London-Don Mills, Ont., 1973. Sorting and searching, Addison-Wesley Series in Computer Science and Information Processing.

[16] Adeline Pierrot and Dominique Rossin. 2-stack sorting is polynomial. In 31st International Symposium on Theoretical Aspects of Computer Science, volume 25 of LIPIcs. Leibniz Int. Proc. Inform., pages 614-626. Schloss Dagstuhl. Leibniz-Zent. Inform., Wadern, 2014.

[17] Rodica Simion and Frank W. Schmidt. Restricted permutations. European J. Combin., 6(4):383-406, 1985.

[18] Wikipedia. Enumerations of specific permutation classes — Wikipedia, the free encyclopedia, 2014. http://en.wikipedia.org/wiki/Enumerations_of_specific_ permutation_classes Accessed 5 July 2014. 